\title{
Tract-Based Spatial Statistics Analysis of Diffusion- Tensor Imaging Data in Patients with Small Cell Lung Cancer.
}

\author{
Benezi $\mathrm{S}^{1,2}$, Karavasilis E ${ }^{3}$, Karanasiou I.S', Matsopoulos $\mathrm{G}^{1}$, Ventouras $\mathrm{E}^{4}$, Uzunoglu N ${ }^{1}$, Kouloulias V ${ }^{5}$, Papathanasiou \\ $\mathrm{M}^{5}$, Foteineas $\mathrm{A}^{5}$, Soldatos $\mathrm{Th}^{3}$, Iosif $\mathrm{Ch}^{3}$, Efstathopoulos $\mathrm{E}^{5}$, Kelekis N ${ }^{5}$, Kelekis D³. \\ 1. National Technical University of Athens School of Electrical \& Computer Engineering Microwave \& Fiber Optics Laboratory. \\ 2. School of Medicine, University of Patras. \\ 3. Research Centre of Radiology and Imaging, "Evgenidion” General Hospital. \\ 4. Department of Medical Instrumentation Technology, Technological Educational Institution of Athens. \\ 5. National and Kapodistrian University of Athens, Division of Radiology-Radiotherapy II
}

\begin{abstract}
Prophylactic cranial irradiation (PCI) is known to improve survival to a significant degree in Small Cell Lung Cancer (SCLC) patients. The aim of our study is to investigate changes in structural connectivity between healthy-volunteers and patients with SCLC, before and after PCI treatment. In this preliminary study we use diffusion tensor imaging (DTI) to identify changes in white matter structure in patient group pre radiotherapy to control group. The results have showed alterations in white matter structure. In the patient group lower fractional anisotropy (FA) and higher eigenvalues in white matter regions are observed compared to the control group. The results presented herein are subject to further investigation with larger patient and control groups.
\end{abstract}

Keywords-component; Diffusion Tensor Imaging; DTI; TractBased Spatial Statistics; TBSS; Small Cell Lung Cancer; SCLC; Prophylactic Cranial Irradiation; PCI; Fractional Anisotropy; FA;

\section{INTRODUCTION}

Small cell lung cancer (SCLC) has a very poor prognosis when untreated. The use of chemotherapy, with or without chest radiotherapy, results in survival improvement and a small percentage of cures. However the majority of the patients relapse and only $<25 \%$ of complete responders become longterm survivors $[1,2,3]$. At the time of initial diagnosis, $10 \%$ to $14 \%$ of patients with SCLC have detectable brain metastases. At the time of death, at least one-third (33\% to $42 \%$ ) of patients present clinically recognized brain metastases, and approximately $50 \%$ of patients have brain metastases when sent to postmortem examination. The frequency of brain metastases increases as survival time increases [1, 2, 3, 4].

Prophylactic cranial irradiation (PCI) has become a standard of care for selected patients with limited and extensive SCLC showing positive outcome with systemic treatment. The main goal of PCI is to eliminate any possible microscopic parts of metastatic tumor in the brain before they become clinically manifest. Without PCI, brain metastases will eventually occur in more than $60 \%$ of small cell lung cancer patients and PCI reduces this rate to approximately $20 \%$ [6]. In parallel, PCI greatly prolongs life expectancy both in the case of limited and extensive disease $[2,5,6]$. As these patients are at high risk for cognitive deficits from multiple causes, such as chemotherapy effects, there has been much controversy in the medical community regarding the extent to which PCI contributes to observed neurocognitive disorders [1-6].

White matter (WM) is recognized as the element in the brain that is most vulnerable to irradiation. Postmortem studies showed a broad spectrum of radiation induced lesions, ranging from vascular damage to coagulation necrosis [7, 8]. The response of cerebral tissue to radiation is dynamic, $[9,10]$ and extending beyond the targeted tumor volume affecting brain neural structures directly and indirectly. Clinical and neurological symptoms can occur within minutes after radiation therapy and up to 30 years after completion of treatment [11]. The most significant neurotoxic effects of radiation is demyelination, inflammation and breakdown of the blood-brain barrier $[9,10,11]$. Dysfunction in cerebrum white matter including the corpus callosum can lead to deficits in sensory and neurocognitive functions $[10,11]$.

Diffusion tensor imaging (DTI) is a non-invasive MRI technique that is very sensitive to white matter structure than conventional MRI $[12,13]$. DTI measures the diffusion of water molecules in the brain, which varies depending on the direction, density and myelination of white matter fibers [13, 14]. Typical parameters of the DTI are mean diffusivity (MD) and fractional anisotropy (FA), which define the magnitude of diffusion and the preferential directionality of water diffusion along the white matter tracts, respectively $[14,15]$. FA reflects overall white matter density and integrity, and decreased FA has been seen in many brain pathologies. Recently, a technique that permits voxelwise statistical analysis of DTI data using tract based spatial statistics (TBSS) has been introduced enabling robust assessment of local differences in white matter integrity between groups. It provides a powerful and objective method for detecting variation on major white matter tract $[16$, 17].

The overall scope of our current research is to investigate anatomical and functional connectivity in SCLC patients as well as their neurocognitive functionality, depending on the 
type of disease and the phase of treatment and time of clinical examination [18]. Our goal in the present study is to investigate potential differentiations in DTI images in SCLC patients before PCI compared to the healthy group. Thus, in this preliminary study, we aim to employ TBSS to test the voxelwise differences in fractional anisotropy (FA), mean diffusivity (MD) and the principal diffusivities $\lambda 1, \lambda 2$ and $\lambda 3$ between healthy-volunteers and patients with SCLC, before PCI.

\section{METHODS}

\section{A. Subjects}

Eleven healthy participants (five females, age range 45-65 years) and eleven SCLC patients (four females, age range 4565 years) before PCI treatment participated in the study. All participants had complete response to initial treatment (chemotherapy with or without chest radiotherapy), without any brain metastases shown, met the standard MRI safety criteria and had no history of diagnosed neurological disorder, major psychiatric disorder or treatment with psychotropic medication, including substance misuse. All participants provided written informed consent and the study was approved by the appropriate research ethics committee.

\section{B. Data Acquisition}

Whole brain MRI, DTI and fMRI data were collected on a Philips 3.0T scanner (Achieva; Philips, Best, The Netherlands) at the Radiology Research Unity, Medical Imaging Department, Evgenidion Hospital, National and Kapodistrian University, Athens, Greece using an 8-channel SENSE head coil used for radiofrequency reception of the nuclear magnetic resonance signals. Foam pads and headphones were used to reduce head motion and scanner noise.

Diffusion tensor data were acquired using a diffusionweighted single-shot spin-echo-planar (SE-EPI) imaging sequence. The acquisition parameters were repetition time $=4156 \mathrm{~ms}$; echo time $=69 \mathrm{~ms}$; flip angle $=900$; acquisition matrix $=128 \times 128$; field of view $=256 \mathrm{~mm}$; slice thickness $=2$ $\mathrm{mm}$, gap $=0,75$ contiguous axial slices. Diffusion-sensitizing gradients were applied along 60 non-collinear directions $(\mathrm{b}=1000 \mathrm{~s} / \mathrm{mm} 2)$, and an acquisition without diffusion weighting $(b=0)$. A high resolution $3 \mathrm{D}$ gradient echo $\mathrm{T} 1$ weighted anatomical data were also acquired $(1.0 \mathrm{~mm}$ slice thickness, $\mathrm{TE}=4.6 \mathrm{~ms}, \mathrm{TR}=15 \mathrm{~ms}, \mathrm{FOV}=256$ and $1 \mathrm{x} 1 \mathrm{x} 1 \mathrm{~mm} 3$ reconstructed voxel size).

\section{Preprocessing and data analysis}

All data were processed using FSL (FMRIB's Software Library, version 5.0; www.fMRIb.ox.ac.uk/fsl) [17]. First of all, every data set was corrected for stretches and shears induced by eddy currents in the gradient coils and simple head motions by using affine transformation of each diffusion weighting image to the reference volume without diffusion. Next, images were brain-extracted using BET (Brain Extraction Tool), part of the FSL package [17]. Finally, a diffusion tensor model was fitted on the data to determine the level of anisotropy for each voxel independently by calculating the tensor eigenvalues describing the diffusion strength in the primary, secondary and tertiary diffusion directions. Fractional anisotropy, $\lambda 1, \lambda 2$ and $\lambda 3$ maps were generated using FDT-FMRIB's Diffusion Toolbox [19]. The FA, a quantification of how strongly directional the local tract structure is, was calculated and plotted in a single FA map for each subject.

Voxel-wise statistical analysis of the FA data was performed using TBSS implemented in FSL [16]. Statistics in TBSS was carried out using the "Randomise" tool. Randomise uses a general linear model and contrasts to test effects of interest with different statistical measures. TBSS tool aligns every FA image to every other one, identifies the "most representative" one and uses this as the target image. Next, all subjects' FA data were aligned into the MNI-152 standard space using a non-linear registration algorithm. The mean FA image was then created and thinned to generate a mean FA skeleton which represented the centers of all tracts common to the group. This was thresholded to $F A \geq 0.2$ to include the major white matter pathways but exclude peripheral tracts where there was significant inter-subject variability and/or partial volume effects with grey matter. Each subject's aligned FA data were then projected onto this skeleton and the resulting data were fed into voxel-wise cross-subject statistics, applying a control $>$ patient $t$ test 1 and a patient $>$ control $t$ test 2 . Maps of $\mathrm{t}$ values were created using threshold-free cluster enhancement (TFCE) to reduce noise [20], and correction for multiple comparisons was achieved using a nonparametric permutation test with 5000 permutations [21], Significance level was set at 0.05 . TBSS analysis was also applied to the $\lambda 1, \lambda 2$ and $\lambda 3$ maps, as well as in MD.

\section{RESULTS}

The results suggest that regions within the centrum semiovale white matter (superior frontal gyrus, limbic lobe, inferior frontal gyrus, inferior temporal gyrus, anterior and posterior limb of internal capsule and superior parietal lobe), the cerebellum and the fornix may present lower FA in SCLC patients compared to controls (voxelwise thresholding uncorrected for multiple comparisons, $\mathrm{t}>3, \mathrm{p}<0.05)$. The results with significant differences in FA, $\lambda 1, \lambda 2, \lambda 3$ and MD maps between the two groups used in the analysis can be seen in Figure 1, where all t maps are displayed highlighted for easier visualization. The MNI152 standard-space image is presented in the background in greyscale with the mean FA skeleton in green. On top of the skeleton are the areas (or clusters) of significant differences between the patient and control group, and in this particular case the significant areas represent clusters where patient group mean FA value is significantly decreased compared to control group mean FA values. Red color indicates the level of significance. Areas where FA values were significantly higher for patients than for controls were not found. TBSS analysis, also showed that regions with FA decreases were accompanied by increased $\lambda 1, \lambda 2, \lambda 3$ and MD (Figure 1). Areas, where significant higher $\lambda 1, \lambda 2, \lambda 3$ and MD values were present in the patients compared to the control group, are visualized with blue, purple, yellow and cool coloring respectively.

Cluster information was extracted from the images, and 20 different clusters were found with the p-value less than 0.02 , as 
shown in TABLE I. These clusters indicate extensive areas with significant differences.

TABLE I. SIGNIFICANT CLUSTERS

\begin{tabular}{|c|c|c|c|c|}
\hline Cluster & Max 1-p & $\begin{array}{c}\text { Max x } \\
\text { (Voxels) }\end{array}$ & $\begin{array}{c}\text { Max y } \\
\text { (Voxels) }\end{array}$ & $\begin{array}{c}\text { Max z } \\
\text { (Voxels) }\end{array}$ \\
\hline 1 & 0.980 & 135 & 66 & 67 \\
\hline 2 & 0.985 & 113 & 66 & 125 \\
\hline 3 & 0.994 & 71 & 144 & 129 \\
\hline 4 & 0.984 & 60 & 97 & 83 \\
\hline 5 & 0.987 & 44 & 133 & 51 \\
\hline 6 & 0.987 & 78 & 175 & 101 \\
\hline 7 & 0.994 & 74 & 179 & 87 \\
\hline 8 & 0.990 & 45 & 70 & 66 \\
\hline 9 & 0.992 & 62 & 120 & 51 \\
\hline 10 & 0.982 & 35 & 101 & 59 \\
\hline 11 & 0.993 & 45 & 110 & 52 \\
\hline 12 & 0.984 & 69 & 160 & 100 \\
\hline 13 & 0.987 & 141 & 72 & 67 \\
\hline 14 & 0.993 & 101 & 131 & 133 \\
\hline 15 & 0.987 & 81 & 114 & 137 \\
\hline 16 & 0.980 & 74 & 160 & 110 \\
\hline 17 & 0.982 & 84 & 58 & 107 \\
\hline 18 & 0.981 & 105 & 162 & 106 \\
\hline 19 & 0.981 & 138 & 70 & 78 \\
\hline 20 & 0.986 & 119 & 153 & 60 \\
\hline
\end{tabular}

\section{Discussion AND CONCLUSION}

In this study, we found some white matter tract damages in patients with SCLC before PCI treatment. The results show decreased FA in superior frontal gyrus, limbic lobe, inferior frontal gyrus, inferior temporal gyrus, anterior and posterior limb of internal capsule, superior parietal lobe and cerebellum. These findings, is likely to represent greater vulnerability to the toxic effects of chemotherapy in specific brain structures. We also analyzed three eigenvectors of the diffusion tensor and the MD. Our results showed that regions with FA decreases were accompanied by increased $\lambda 2$ and $\lambda 3$ (i.e., increased radial diffusivity). While the $\lambda 1$, which contributes to axial diffusivity and reflects the number and diameter of axons, exhibit smaller changes. The same is observed for mean diffusivity, lesser increase than in the case of $\lambda 1$ and $\lambda 2$. These results seem to be in line with studies where increased RD has been correlated to decreased myelination in several models of brain injury, including radiation-induced demyelination [22, 23, 24, 25].

Previous studies have demonstrated regional variation in white matter diffusion index changes following chemotherapy and radiotherapy, with larger changes implying greater white matter degradation. Large changes in the inferior cingula and fornix have implications for predicting and preventing chemoradiotherapy induced neurocognitive impairment [22]. A significant reduction in anisotropy (FA) is found in cerebellar, frontal WM, and corona radiata in patients with SCLC after PCI [7].
This article presents the initial results of a much larger study, which is ongoing. The purpose of this research is to investigate changes in structural connectivity between healthyvolunteers and patients with SCLC, before and after PCI treatment. Our aim is to contribute to the current knowledge whether radiation contributes to the observed neurocognitive disorders. Anatomical and functional connectivity in healthy subjects and patients with cancer (resting state networks) [18], depending on the type of disease and the phase of treatment and time of clinical examination, will be studied. Study of cognitive functionality of patients with SCLC will be conducted with imaging and clinical examination. The effects of radiation (PCI) both at anatomical as well as functional levels in the brain will be consequently assessed. Finally, main objective of the present investigation is to unveil aspects of the neuropathophysiology of the disease, aiming at more accurate presymptomatic diagnosis and adaptation of treatment management.

This is the first study to explore white matter injury in patients with SCLC by using TBSS for DTI data analysis. TBSS, as an objective and sensitive method, can reveal multiple white matter microstructural abnormalities in patients (SCLC) following chemoradiotherapy. To the best of our knowledge this kind of analysis has not been previously reported. It should be noted though that the preliminary results presented herein are subject to further investigation with larger patient and control group. In the near future, we plan to perform analysis of DTI images before and after PCI treatment in SCLC patients.

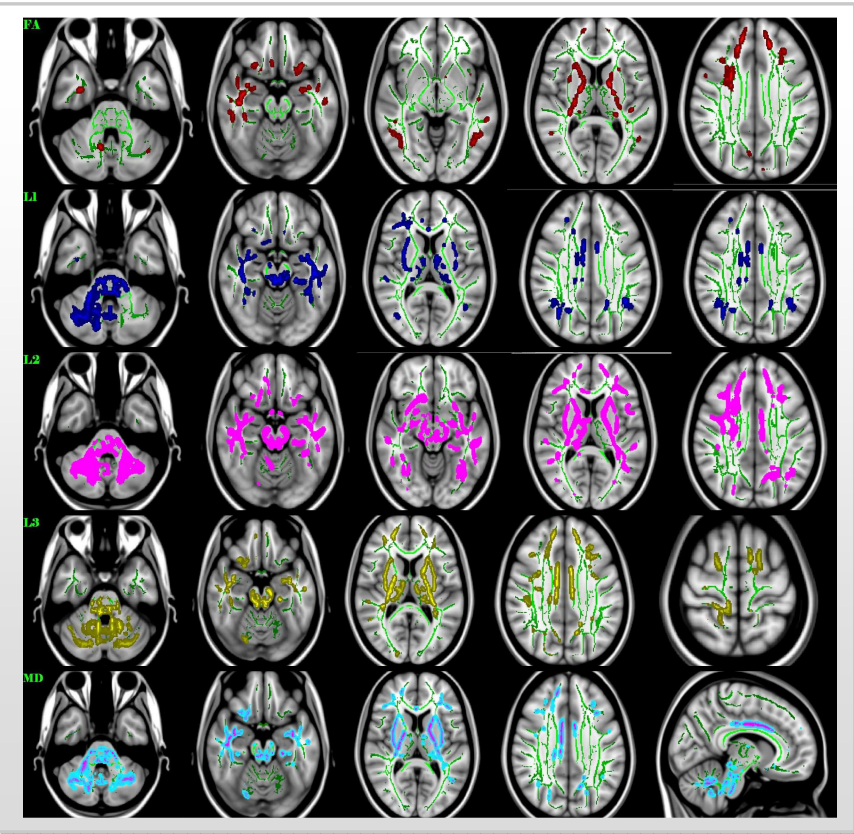

Figure1. Five sample images of the qualitative results from TBSS analysis for the used patient and control database. Significant differences, where patient FA is lower than control FA, are visualized with red coloring and the increase of $\lambda 1, \lambda 2, \lambda 3$ and MD are visualized with blue, purple, yellow and cool respectively. Mean FA skeleton is presented in green color. All t maps are displayed highlighted for easier visualization. The first row shows differences in FA, the second in $\lambda 1$, the third in $\lambda 2$, the fourth in $\lambda 3$ and the last row in MD. 


\section{ACKNOWLEDGMENT}

This research has been co-financed by the European Union (European Social Fund - ESF) and Greek national funds through the Operational Program "Education and Lifelong Learning" of the National Strategic Reference Framework (NSRF)-Research Funding Program: THALIS - NTUA, "Study and Analysis of Medical Data using structural and functional Magnetic Resonance Imaging procedures (MRI/DTI/fMRI): Assessment of changes induced by Brain Radiotherapy" (MIS 380151).

\section{References}

[1] R. Arriagada, I. Monnet, A. Riveiere et al. "Prophylactic cranial irradiation for patients with small cell lung cancer in complete response,” Eur J Cancer 1995; 31A (suppl 5): S19.

[2] A. Auperin, R. Arriagada, J-P Pignon et al. "Prophylactic cranial irradiation for patients with small-cell lung cancer in complete remission," N Engl J Med 1999; 341:476-484

[3] G. Y. Yang, R. H. Matthews "Prophylactic Cranial Irradiation in SmallCell Lung Cancer,” The Oncologist 2000; 5:293-298.

[4] AP. Meert, M. Paesmans, T. Berghmans, B. Martin, C. Mascaux et al. "Prophylactic cranial irradiation in small cell lung cancer: a systematic review of the literature with meta-analysis," BMC Cancer 2001; 1: 5.

[5] B. Slotman, C. Faivre-Finn, G. Kramer, et al., "Prophylactic cranial irradiation in extensive small-cell lung cancer," New England Journal of Medicine, vol. 357, no. 7, pp. 664-672, 2007.

[6] J. C. Marsh, B. T. Gielda, A. M. Herskovic, and R. A. Abrams "Cognitive Sparing during the Administration of Whole Brain Radiotherapy and Prophylactic Cranial Irradiation: Current Concepts and Approaches," Journal of Oncology 2010; Article ID 198208, 16 pages.

[7] T. Welzel, A. Niethammer, U. Mende, S. Heiland, F. Wenz, J. Debus, R. Krempien, "Diffusion Tensor Imaging Screening of Radiation-Induced Changes in the White Matter after Prophylactic Cranial Irradiation of Patients with Small Cell Lung Cancer: First Results of a Prospective Study,” AJNR Am J Neuroradiol 2008; 29:379-83.

[8] PE Valk, WP Dillon. "Radiation injury of the brain," AJNR Am J Neuroradiol 1991;12:45-62.

[9] TE Schultheiss, LE Kun, KK Ang, et al. "Radiation response of the central nervous system," Int J Radiat Oncol Biol Phys 1995; 31:10931112 .

[10] PJ Tofilon, JR Fike. "The radioresponse of the central nervous system: a dynamic process," Radiat Res 2000; 153:357-370.

[11] V Nagesh, CI Tsien, TL Chenevert, et al. "Radiation-induced changes in normal-appearing white matter in patients with cerebral tumors: a diffusion tensor imaging study," Int J Radiat Oncol Biol Phys. 2008; 70:1002-1010

[12] Y. Assaf, O. Pasternak "Diffusion tensor imaging (DTI)-based white matter mapping in brain research: a review," J Mol Neurosci 2008; 34: 51-61. doi: 10.1007/ s12031-007-0029-0.

[13] CH. Chapman, M. Nazem-Zadeh, OE. Lee, MJ. Schipper, CI. Tsien, TS. Lawrence, Y. Cao "Regional Variation in Brain White Matter Diffusion
Index Changes following Chemoradiotherapy: A Prospective Study Using Tract-Based Spatial Statistics," PLOS ONE, 2013 MAR 4; 8 (3): -

[14] D. Le Bihan, JF. Mangin, C. Poupon, CA. Clark, S. Pappata, N. Molko, H. Chabriat "Diffusion tensor imaging: Concepts and applications," J Magn Reson Imaging 2001; 13:534-546.

[15] R. Aliotta, J. L Cox, K. Donohue, B. Weinstock-Guttman, E A. Yeh, P. Polak, M. G. Dwyer, R. Zivadinov "Tract-Based Spatial Statistics Analysis of Diffusion- Tensor Imaging Data in Pediatric- and AdultOnset Multiple Sclerosis,” Hum Brain Mapp 2014 Jan 30; 35(1):53-60. Epub 2012 Aug 30.

[16] S.M. Smith, M. Jenkinson, H. Johansen-Berg, D. Rueckert, T.E. Nichols, C.E. Mackay, K.E. Watkins, O. Ciccarelli, M.Z. Cader, P.M. Matthews, and T.E.J. Behrens. "Tract-based spatial statistics: Voxelwise analysis of multi-subject diffusion data," NeuroImage, 31:1487-1505, 2006.

[17] S.M. Smith, M. Jenkinson, M.W. Woolrich, C.F. Beckmann, T.E.J. Behrens, H. Johansen-Berg, P.R. Bannister, M. De Luca, I. Drobnjak, D.E. Flitney, R. Niazy, J. Saunders, J. Vickers, Y. Zhang, N. De Stefano, J.M. Brady, and P.M. Matthews. "Advances in functional and structural MR image analysis and implementation as FSL," NeuroImage, 23(S1):208-219, 2004.

[18] K. Bromis, I. S. Karanasiou, G. Matsopoulos, E. Ventouras, N. Uzunoglu, G. D. Mitsis, E. Karavasilis, M. Papathanasiou, N. Kelekis and V. Kouloulias, "Resting state and task related fMRI in small cell lung cancer patients," (2013) 13th IEEE International Conference on BioInformatics and BioEngineering, IEEE BIBE 2013, art. no. 6701662 DOI: 10.1109/BIBE.2013.6701662.

[19] Behrens, T.E., Woolrich, M.W., Jenkinson, M., Johansen-Berg, H., Nunes, R.G., Clare, S., Matthews, P.M., Brady, J.M., Smith, S.M. "Characterization and propagation of uncertainty in diffusion-weighted MR imaging," Magn. Reson. Med. 2003; 50, 1077-1088.

[20] Smith SM, Nichols TE "Threshold-free cluster enhancement: Addressing problems of smoothing, threshold dependence and localisation in cluster inference," Neuroimage 2009; 44: 83-98. doi:10.1016/j.neuroimage.2008.03.061.

[21] Nichols TE, Holmes AP. "Nonparametric permutation tests for functional neuroimaging: a primer with examples," Hum Brain Mapp 2002; 15: 1-25.

[22] C H. Chapman, M. Nazem-Zadeh, O. E. Lee, M. J. Schipper, C. I. Tsien1, T. S. Lawrence, Y. Cao "Regional Variation in Brain White Matter Diffusion Index Changes following Chemoradiotherapy: A Prospective Study Using Tract-Based Spatial Statistics," Plos One 2013; Volume 8, Issue 3 .

[23] CL. Mac Donald, K. Dikranian, P. Bayly, D. Holtzman, D. Brody "Diffusion Tensor Imaging Reliably Detects Experimental Traumatic Axonal Injury and Indicates Approximate Time of Injury," Journal of Neuroscience 27: 11869-11876. doi:10.1523/jneurosci.3647-07.2007.

[24] S. Wang, EX. Wu, D. Qiu, LHT. Leung, H-F. Lau, et al. "Longitudinal diffusion tensor magnetic resonance imaging study of radiation-induced white matter damage in a rat model," Cancer Res (2009) 69: 1190-1198. doi:10.1158/0008-5472.CAN-08-2661.

[25] M. Anjari, L. Srinivasan, JM. Allsop, JV. Hajnal, Rutherford, MA. Edwards AD, SJ. Counsell "Diffusion tensor imaging with tract-based spatial statistics reveals local white matter abnormalities in preterm infants," Neuroimage. 2007 Apr 15;35(3):1021-7. Epub 2007 Feb 8. 